UVX 2008 (2009) 57-63

(C) EDP Sciences, 2009

DOI: $10.1051 / \mathrm{uvx} / 2009010$

\title{
LASERIX : premier bilan du fonctionnement de l'installation
}

\author{
O. Guilbaud ${ }^{1}$, D. Ros ${ }^{1}$, S. Kazamias ${ }^{1}$, B. Zielbauer ${ }^{1}$, J. Habib ${ }^{1}$, M. Pittman ${ }^{1}$, \\ K. Cassou ${ }^{1}$, F. Plé ${ }^{1}$, M. Farinet ${ }^{1}$, A. Klisnick ${ }^{2}$, F. de Dortan ${ }^{2}$, S. Lacombe ${ }^{4}$, \\ E. Porcel ${ }^{4}$, C. Le Sech ${ }^{4}$, M.-A. du Penhoat ${ }^{5}$, A. Touati ${ }^{5}$, M. Marsi ${ }^{8}$, \\ M. Fajardo ${ }^{7}$, P. Zeitoun ${ }^{6}$ et D. Joyeux ${ }^{3}$ \\ ${ }^{1}$ LASERIX, CLUPS, Univ. Paris-Sud, Orsay, France \\ ${ }^{2}$ LIXAM, Univ. Paris-Sud, CNRS, Bât. 350, Campus d'Orsay, 91405 Orsay Cedex, France \\ ${ }^{3}$ LCFIO, Univ. Paris-Sud, CNRS, Campus Polytechnique, RD 128, 91127 Palaiseau Cedex, \\ France \\ ${ }^{4}$ LCAM, Univ. Paris-Sud, CNRS, Bât. 351, Campus d'Orsay, 91405 Orsay Cedex, France \\ ${ }_{5}^{5}$ IMPMC, Univ. Paris VII, CNRS, Campus de Jussieu, 75000 Paris, France \\ ${ }^{6}$ LOA, ENSTA, CNRS, Chemin de la Hunière, 91761 Palaiseau Cedex, France \\ ${ }^{7}$ GolP, Instituto Superior Técnico, Av. Rovisco Pais 1049-001 Lisboa, Portugal \\ ${ }^{8}$ LPS, Univ. Paris-Sud, CNRS, Bât. 510, Campus d'Orsay, 91405 Orsay Cedex, France
}

\begin{abstract}
Résumé. The LASERIX facility provides coherent and short soft x-ray beams for scientific applications. The beams are generated through high intensity laser interaction with matter using two different schemes, plasma based soft x-ray lasers, and high order laser harmonic generation. We describe in this communication the present status of the facility. The experimental setup composed of a terawatt titanium-sapphire laser and of different IR/XUV conversion devices is briefly described. The LASERIX beamtime has been recently opened to external users. We present two typical experiments performed in that context with the facility. The first one is dedicated to the fundamental study of the plasma based soft $\mathrm{x}$-ray laser, whereas the second uses the existing beam to study irradiation induced damages in DNA samples. The future evolution of the facility, including enhancement of the laser energy is finally presented.
\end{abstract}

\section{INTRODUCTION}

Le développement des lasers intenses a permis d'accéder à de nouveaux régimes d'interaction de la lumière avec la matière. Les intensités en jeu donnent naissance à des milieux ionisés (plasmas), caractérisés par de hautes densités d'énergie, des états loin de l'équilibre thermodynamique, et qui sont le siège de phénomènes hautement non-linéaires. Certains processus conduisent à une conversion d'une partie de l'énergie laser en rayonnements électromagnétiques de fréquences très différentes de l'onde incidente, ou en faisceaux de particules suprathermiques (électrons, protons, ions, neutrons...) [1]. Ces faisceaux peuvent présenter des propriétés originales en terme de brièveté et de brillance. Après une phase d'exploration et d'étude, commence maintenant une phase d'optimisation et d'utilisation de ces nouvelles sources. Quelques projets d'installations laser dédiées ont ainsi vu le jour. le projet LASERIX s'inscrit dans cette démarche mais se distingue par son ampleur.

LASERIX est un projet de l'université Paris-Sud financé par le conseil général de l'Essonne dans le cadre du Contrat Plan Etat-Région 2002-2006 [2]. Il s'agit d'une installation expérimentale qui délivre des faisceaux de rayonnement XUV $(10 \mathrm{~nm}<\lambda<40 \mathrm{~nm})$ aux propriétés originales pour des applications scientifiques. Ces sources reposent sur la conversion de rayonnement laser infrarouge intense en rayonnement de courtes longueurs d'onde par interaction laser-matière. Le laser infrarouge, de type titane-saphir, sera décrit dans la première partie de ce papier. Les deux schémas de conversion, le laser X d'une part et les harmoniques laser d'ordre élevé d'autre part, seront également présentés. La vocation de cette installation est double. Il s'agit de permettre à des chercheurs extérieurs à l'installation de mener à bien des expériences avancées sur la physique et la technologie des sources XUV produites 
par laser. La disponibilité et la flexibilité de l'installation seront ici des maîtres mots. Il s'agit par ailleurs de mettre à la disposition d'une communauté plus vaste, des sources pertinentes, optimisées, fiables et stables pour permettre l'émergence de nouvelles applications en physique du solide, en biologie, chimie ... Nous présenterons et commenterons un exemple de chacun de ces types d'expériences réalisé récemment sur la station LASERIX. Cette dernière est ouverte aux utilisateurs extérieurs depuis janvier 2008. Cette première phase de fonctionnement a permis de valider le concept général du projet tout en mettant en évidence un certain nombre de points où des progrès sont encore à accomplir. A partir de decembre 2008, la station va être déménagée dans un nouveau bâtiment plus vaste pour que son potentiel puisse être pleinement exploité. Nous donnerons en guise de conclusion un apercu de cette évolution programmée.

\section{DESCRIPTION DE I'INSTALLATION}

\subsection{Le laser infrarouge}

La station LASERIX est installée au Laboratoire d'Optique Appliquée, dans les locaux de l'ENSTA à Palaiseau. Le moteur de cette installation est une chaîne laser CPA à oscillateur et amplificateur titanesaphir. Cette installation délivre dans sa version actuellement opérationnelle des impulsions infrarouges (longueur d'onde centrale de $800 \mathrm{~nm}$ ) de $2 \mathrm{~J}$ à une cadence de $10 \mathrm{~Hz}$ et recomprimables à $35 \mathrm{fs}$. Après le dernier étage amplificateur, le faisceau est séparé et distribué sur trois voies différentes (voir figure 1). Dans la première voie dite «nanoseconde», une fraction importante (40\%) de l'énergie est utilisée directement sous sa forme non comprimée. La durée de l'impulsion est alors de 500 ps. Un dispositif installé sur cette voie permet de modifier la forme temporelle de l'impulsion en la faisant précéder d'une pré-impulsion d'énergie et de délai variables. L'autre fraction est envoyée dans un compresseur haute énergie (sous-vide) pour être comprimée en une impulsion de durée comprise entre 35 fs et une dizaine de picosecondes selon les besoins. Cette deuxième voie laser est pour cette raison appelée voie «picoseconde». Ces deux premières voies seront utilisées pour générer un laser XUV à plasma (voir ci-dessous). La troisième voie laser de l'installation provient d'un prélèvement de quelques pourcents de l'énergie laser en sortie d'amplification. Elle est comprimée dans un petit compresseur pour donner des impulsions de $35 \mathrm{fs}$ de durée et de $20 \mathrm{~mJ}$ d'énergie. Les impulsions de cette voie "femtoseconde" sont employées pour générer des harmoniques laser d'ordre élevée.

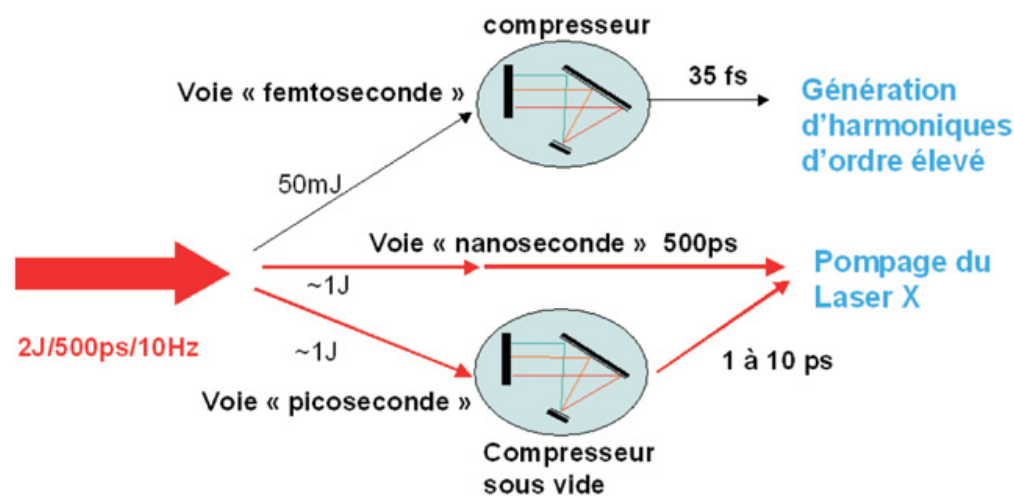

Figure 1. Distribution de l'énergie laser après le dernier étage d'amplification.

De part sa conception l'installation peut donc délivrer trois faisceaux lasers (voire plus) d'énergies, de durée, de forme temporelle variables, parfaitement synchronisées et vers un même système 
d'enceintes sous vide. Ces faisceaux peuvent être employés pour des expériences pompes-sondes IR-XUV ou pour réaliser des sources XUV à plusieurs étages.

\subsection{Les sources XUV}

Deux méthodes sont utilisées pour générer, à partir du laser infrarouge, des faisceaux XUV possédant différentes propriétés de cohérence. La première méthode de conversion consiste à focaliser le faisceau "femtoseconde" dans une cellule contenant un gaz rare (Argon ou Néon) [3]. L'intensité du champ laser au point focal atteint des ordres de grandeurs comparables au champ qu'exercent les atomes du gaz sur leurs électrons périphériques. Ceci donne lieu à des phénomènes optiques hautement nonlinéaires, conduisant à l'émission de rayonnement totalement cohérent, femtoseconde, à des fréquences harmoniques de la fréquence fondamentale. Ce phénomène étant de nature centrosymétrique, seules les harmoniques d'ordres impaires sont émises. Le dispositif de génération d'harmonique de la station LASERIX a permis de générer des harmoniques dans l'argon et dans le néon. Pour une génération dans l'argon des énergies de l'ordre de quelques $\mathrm{nJ}$ par harmonique ont été obtenues autour de la longueur d'onde $32 \mathrm{~nm}$.

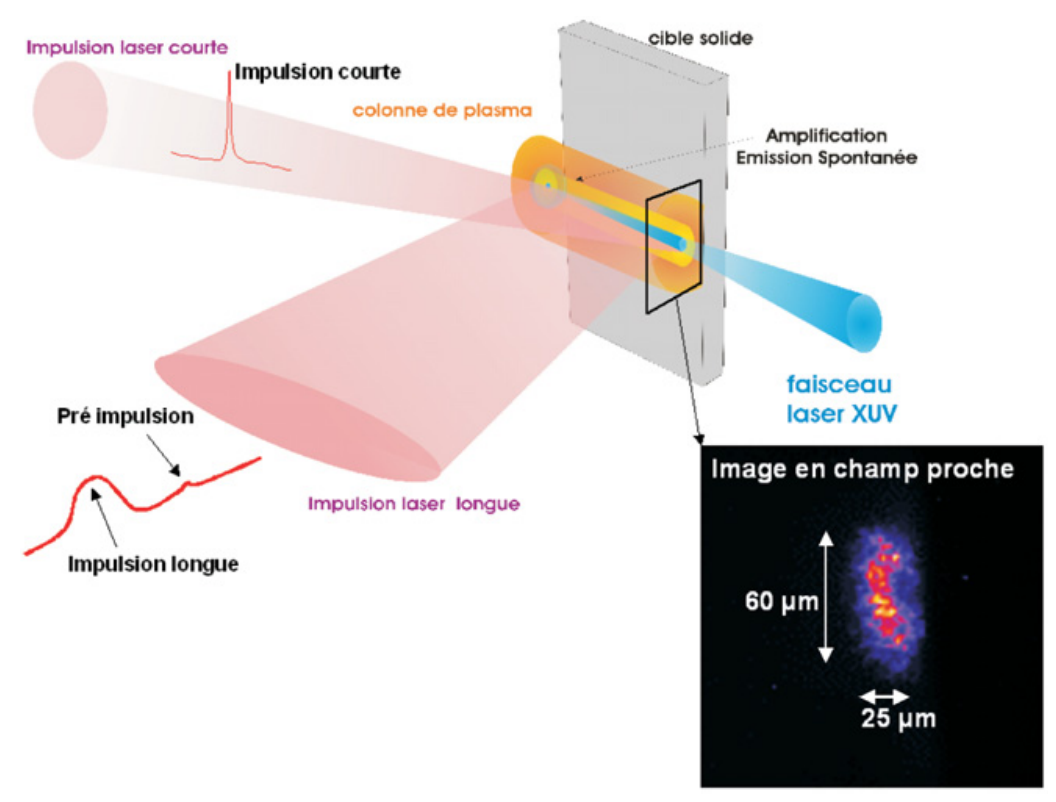

Figure 2. (a) Principe de la source laser X développée sur la station LASERIX. (b) L'encart présente une image en champ proche de la source obtenue avec un imageur XUV à haute résolution.

L'essentielle de l'énergie du laser infrarouge est cependant utilisée pour générer une source de type laser XUV à plasma. Le principe de ce dernier est présenté dans la figure 2 [4,5]. Les faisceaux lasers des voies "nanoseconde" et "picoseconde" sont focalisés sur une cible solide plane rectangulaire de $4 \mathrm{~mm}$ de large. L'impulsion "nanoseconde" génère tout d'abord un tube de plasma riche en ions nickelloïdes. L'impulsion "picoseconde" interagit quelques centaines de picoseconde plus tard avec le plasma en chauffant rapidement les électrons libres. Ceux-ci vont induire par excitation collisionnelle une inversion de population entre les niveaux $4 \mathrm{~d}$ et $4 \mathrm{p}$ des ions nickelloides. Pour optimiser l'efficacité du pompage, le faisceau "picoseconde" arrive sur le plasma avec une incidence élevée par rapport à la normale à la cible. L'émission spontanée émise à une extrémité du plasma est amplifiée lors de sa propagation le long du tube de plasma par le gain laser très élevé ainsi produit. Le faisceau XUV émis en sortie 
du plasma présente une divergence de quelques milliradiants, une faible cohérence spatiale, mais une grande cohérence temporelle (due à la finesse de la raie ionique utilisée) et contient un grand nombre de photons XUV (microjoule). La longueur d'onde d'émission dépend du type d'ions et donc de la composition chimique initiale de la cible. Une cible solide en Molybdène donnera un laser X à 18,9 nm (le laser X couramment utilisé sur l'installation) tandis qu'une cible solide en Argent donnera une émission partiellement cohérente à $13,9 \mathrm{~nm}$. Différents diagnostics sont en place de manière permanente pour caractériser le laser X. La figure $3 \mathrm{~b}$ montre ainsi une image en champ proche de la sortie d'un laser $\mathrm{X}$ à Molybdène obtenue avec un imageur XUV à haute résolution.

\section{FONCTIONNEMENT DE L'INSTALLATION}

\subsection{Généralités}

Après une phase d'installation et de développement du laser infrarouge, les sources XUV ont été validées puis optimisées à partir de l'année 2007. Depuis janvier 2008, LASERIX a débuté un mode de fonctionnement en installation ouverte aux utilisateurs extérieurs. Le temps de faisceau pour chaque type d'expérience est attribué par blocs de deux à trois semaines. Ces périodes sont entrecoupées de périodes de maintenance et de développements internes. Quatre projets ont été accueillis cette année. Deux d'entre eux concernaient la physique des sources XUV tandis que l'autre moitié étaient des expériences d'applications du laser XUV. La liste des thèmes abordés est présentée ci-dessous:

1. irradiation d'échantillons biologiques (responsable: Sandrine Lacombe, LCAM, Orsay)

2. effets non-linéaires XUV en physique du solide (responsable: Marino Marsi, LPS, Orsay)

3. largeur spectrale d'un laser XUV en mode ASE ou en mode injecté par des harmoniques (responsable: Annie Klisnick, LIXAM, Orsay)

4. determination de la fluence de saturation d'un laser XUV injecté par des harmoniques (Responsable: Marta Fajardo, IST, Lisbonnes dans le cadre du consortium européen TUIXS)

Pendant le temps de développement en interne, des travaux ont été menés sur la focalisation des différentes sources XUV ainsi que sur le développement d'un autocorrélateur mono-coup pour les lasers infrarouges femtoseconde de très haute énergie.

\subsection{Exemple 1 : mesure de la largeur spectrale d'un laser XUV}

Nous présentons deux des quatres expériences menées sur LASERIX sous la responsabilité d'utilisateurs extérieurs. La largeur spectrale d'un laser X est une grandeur très difficile à mesurer expérimentalement mais qui présente un très grand intérêt. Elle reflète en effet les conditions physiques du plasma amplificateur et détermine par ailleurs la plus petite durée d'impulsion accessible avec ce dernier. La difficulté de sa détermination réside dans sa très faible valeur $\left(\Delta \lambda / \lambda<10^{-4}\right)$. L'expérience proposée par l'équipe d'Annie Klisnick (LIXAM, Univ. Paris-Sud, CNRS) avait pour objectif de mesurer la largeur spectrale pour une méthode interférométrique [6,7]. Une présentation détaillée de celle-ci faisant l'objet de la contribution de J. Habib à cette conférence, nous nous contenterons de souligner les défis qui ont du être relevés pour la mener à bien. La méthode interférométrique nécessite d'effectuer un grand nombre de tirs pour obtenir une mesure précise de la largeur spectrale. Pour effectuer une étude systématique de cette grandeur en fonction de différents paramètres de génération ou de pompage du plasma, une haute cadence de tir est donc indispensable. Il faut par ailleurs garantir une grande stabilité de la source tir à tir ainsi que de faibles dérives du laser de pompe sur un temps comparable à celui de l'expérience. Ces conditions ont pu être remplies et ont permis de mener la première étude exhaustive de la largeur spectrale d'un laser X en fonction de ses différents paramètres de génération. Une telle étude permettra de valider les predictions des codes hydrodynamiques employés pour simuler le laser $\mathrm{X}$ et permettra de plus de trouver les conditions qui maximisent la largeur spectrale, cela afin de tendre vers des impulsions laser X subpicosecondes. 


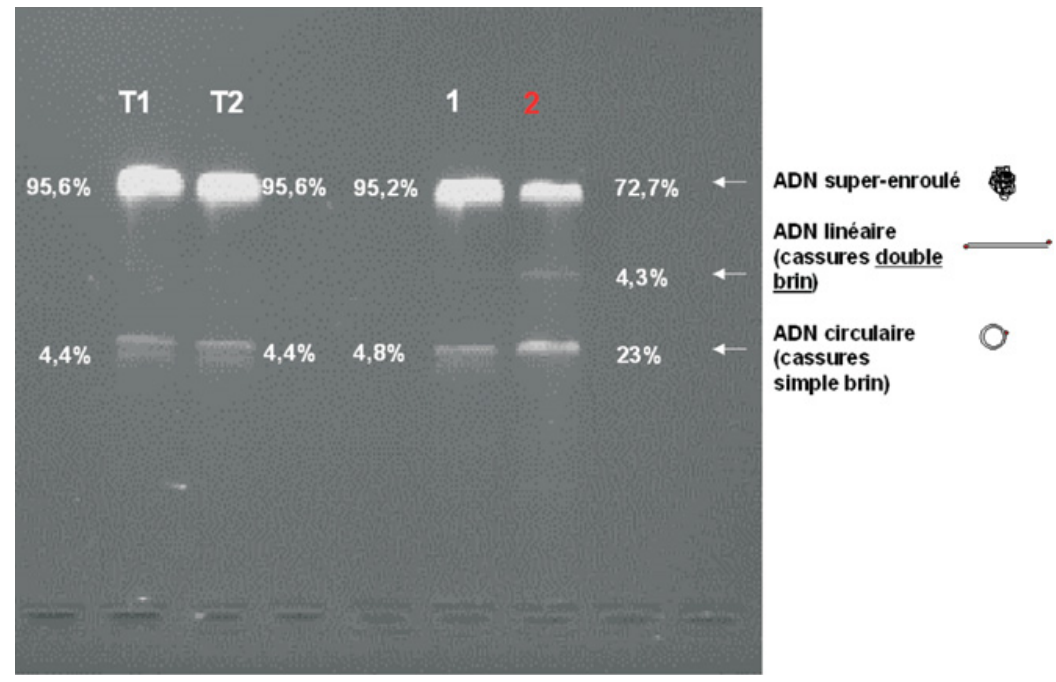

Figure 3. Photographie d'un gel d'électrophorèse après migration. Les échantillons T1, T2, 1 et 2 ont été préparés dans les mêmes conditions. T1 et T2 n'ont pas été exposés au vide. 1 et 2 ont été placés sur la roue porte échantillon, sous-vide. Seul l'échantillon 2 a été irradié par le laser X.

\subsection{Exemple 2 : irradiation d'échantillons biologiques par laser XUV}

L'effet des radiations ionisantes sur l'ADN constitue un vaste sujet d'études compte tenu de son importance en radiothérapie, mais aussi en raison de la variété des mécanismes conduisant à l'endommagement du matériel génétique de la cellule. Si le rôle des électrons secondaires d'énergie inférieure à $1 \mathrm{KeV}$ est maintenant clairement identifié, l'effet de photons dans la même gamme d'énergie est encore largement inexploré. Les sources développées sur LASERIX constituent donc des outils pertinents pour avancer dans ce domaine [8]. L'expérience que nous allons décrire résulte d'une collaboration entre le LCAM (Paris Sud XI, CNRS), l'IMPMC (université paris VII, CNRS) et l'équipe d'accueil de LASERIX. Son objectif était d'irradier sous vide, avec un laser $\mathrm{X}(\lambda=18,9 \mathrm{~nm})$, des échantillons d'ADN sous forme de plasmides lyophilisés. Le faisceau laser X est concentré sur un échantillon de quelques millimètres de diamètre par un miroir sphérique multicouche. Les dommages induits sont analysés a posteriori par une électrophorèse. Selon le type de dommage, les plasmides se trouvent sous une forme super-enroulée (intact), circulaire (cassure d'un brin de la double hélice d'ADN) ou linéaire (cassure double brin) et vont donc migrer différemment sur le gel d'électrophorèse.

La figure 3 présente le résultat d'une électrophorèse des premiers échantillons irradiés. Alors que les échantillons témoins ne présentent qu'un taux faible de cassures simple brin, l'échantillon irradié présente un taux plus élevé de ce type de dommage ainsi qu'un fraction significative de cassures double brin. A court terme cette expérience sera prolongée par une étude systématique des dommages en fonction de la dose et de l'énergie des photons incidents.

Les difficultés propre à cette expérience nous ont conduits à développer certaines techniques utiles pour d'autres applications. La dose à délivrer sur chaque échantillon était très supérieure à la dose contenue dans une seule impulsion laser X. Un minimum de 20000 tirs par échantillon s'est ainsi révélé nécessaire. Nous avons travaillé sur la stabilité de la source afin d'obtenir un niveau d'énergie constant sur ces longues périodes d'irradiation. Par ailleurs, afin d'économiser la cible laser X, nous avons du trouver un régime d'interaction permettant d'effectuer un grand nombre de tirs sur un même emplacement de cible (jusqu'à 300). Comme le montre la figure 4, nous avons observé que l'énergie tir à tir restait relativement stable pendant 200 tirs avant de chuter lentement. L'énergie intégrée par emplacement n'évolue que très lentement au cours du temps. Un réalignement périodique du laser 


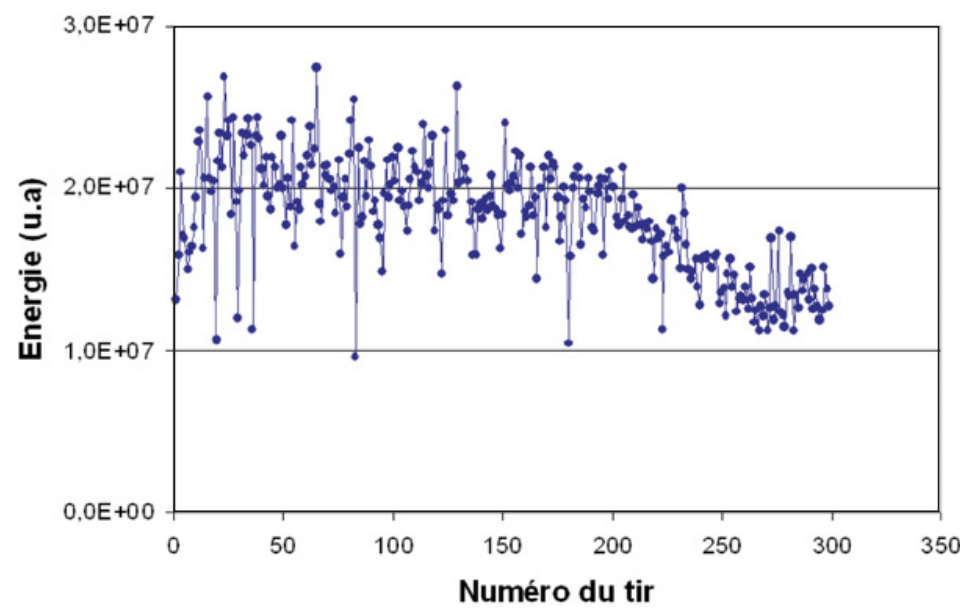

Figure 4. Énergie du laser $\mathrm{X}$ en fonction du nombre de tir sur un même emplacement de la cible.

permet de compenser cette dérive. Enfin nous avons montré qu'un remplacement de cible s'effectuait en moins de 20 minutes (pompage de l'enceinte d'interaction inclu) et qu'aucun réalignement n'était nécessaire pour obtenir à nouveau du laser $\mathrm{X}$.

La dose déposée sur l'échantillon a par ailleurs été mesurée au cours de l'irradiation à l'aide de différents diagnostics calibrés au préalable avec une photodiode XUV étalonnée. Une grille de cuivre placée sur le trajet du faisceau laser X, ainsi qu'une électrode reliée au miroir multicouche de focalisation ont permis de mesurer par effet photoélectrique le nombre de photons émis à chaque tir d'irradiation. Enfin, l'expérience a été installée dans une enceinte dédiée aux applications, équipée de plusieurs diagnostics de visualisation sous vide et la coordination tir laser / déplacement de la cible a été, à cette occasion, automatisée.

\section{CONCLUSIONS ET PERSPECTIVES 2009-2011}

Cette première année d'ouverture aux utilisateurs a permis de valider la pertinence du concept porté par Laserix: développer une installation dédiée à l'exploitation du rayonnement XUV généré par interaction laser intense-matière. Cette confrontation à la réalité nous a permis de mettre en évidence les points qui doivent être impérativement améliorés. La stabilité et la fiabilité du laser ont été à l'origine de problèmes fréquents sur l'installation. Une démarche est actuellement entreprise pour progresser sur ces aspects. Par ailleurs, un dispositif développé en collaboration avec le GSI (Darmstadt, Allemagne) permettra de générer le laser X en n'utilisant qu'un seul faisceau laser au lieu de deux actuellement. Toutes les impulsions lasers nécessaires (longues et courtes) seront générées en amont de la chaîne[10], ce qui simplifiera considérablement le réglage du laser $\mathrm{X}$ et améliorera sa stabilité.

A partir de janvier 2009, l'installation laserix va être démontée et réinstallée dans un nouveau batiment du LOA, afin de disposer d'un environnement plus stable et d'une plus grande surface. La priorité sera donnée à la remise en route du dispositif à $10 \mathrm{~Hz}$ présenté dans cet exposé. Ceci permettra de réouvrir au plus vite l'installation aux utilisateurs. En parallèle sera mené la finalisation du système laser. Un nouvel étage amplificateur et son compresseur associé seront installés. Cet étage, dont le principe a été démontré en 2006 [9], fournira une énergie laser de $40 \mathrm{~J}$ à une cadence de $0,1 \mathrm{~Hz}$. Trois modes de fonctionnement seront utilisés. Dans une premiere phase, le temps entre expériences à $10 \mathrm{~Hz}$ et à $0,1 \mathrm{~Hz}$ sera partagé. Dans un second temps, un dispositif permettra de partager les impulsions en 99 tirs pour une expérience à $10 \mathrm{~Hz}$ et 1 tir pour une expérience nécessitant toute l'énergie. Enfin, une 
partie de l'énergie diffractée dans l'ordre zéro du réseau du compresseur haute énergie sera recyclée pour être comprimée dans le compresseur actuel pour servir de faisceau de pompe ou de sonde.

Une enceinte d'interaction plus grande, prête à être livrée, permettra en outre de disposer de deux zones d'interaction laser-cible. Plusieurs modes d'utilisation peuvent être envisagés:

- une zone pourrait être attribuée au développements de nouvelles sources XUV tandis qu'une autre zone sera réservé à la génération d'un laser X fiabilisé, laissé à demeure pour les applications

- expériences pompe-sonde: Une zone d'interaction servira à générer un plasma, perturber un échantillon, etc ... à l'aide d'un faisceau laser (pompe) tandis qu'un laser X généré dans l'autre zone d'interaction servira de sonde

- un mode multi-étage pourrait finalement être envisagé avec ce même dispositif. Des harmoniques laser sont injectées à l'entrée d'un premier plasma laser X pour y être amplifiées. Le faisceau résultant est ensuite à son tour amplifié dans un plasma laser X plus large. Le principe de la chaine laser serait ainsi transposé dans le domaine XUV.

En parallèle de cette évolution technique, des structures de pilotage de l'installation ainsi qu'un comité d'attribution du temps d'expérience seront mis en place en concertation avec les futurs utilisateurs.

\section{Références}

[1] P. Audebert et al., dans cette conférence.

[2] D. Ros et al., proceedings of the 11th international conference on x-ray lasers, Belfast (2008) to be published.

[3] M. Ferray et al., J. Phys. B 21 (1988) L31.

[4] R. Keenan, J. Dunn et al. Phys. Rev. Lett. 94,(2005) 103901.

[5] K. Cassou et al. Optics Lett. 32 (2006) 139-41.

[6] P. Celliers et al., Optics Lett. 20 (1995) 1907.

[7] A.Klisnick et al., J.Q.S.R.T. 99, (2006) 370.

[8] K. Cassou et al. J. Phys. IV, 127 (2005) 177-180.

[9] F. Ple, M. Pittman, G. Jamelot, and J.-P. Chambaret, Optics Lett., Vol. 32, Issue 3.

[10] D. Zimmer, B. Zielbauer et al., Optics Express 16 (2008)10398-10404. 